\title{
Evaluation of urgent multivisceral resections due to complications resulting from an advanced ovarian cancer
}

\author{
Tomasz Miłek' ${ }^{1}$, Witold Woźniak' , Piotr Porzycki ${ }^{1}$, Alkalayla Habib², Agnieszka Timorek2 \\ Piotr Ciostek ${ }^{1}$, Włodzimierz Sawicki ${ }^{2}$, Krzysztof Cendrowski ${ }^{2}$ \\ ${ }^{1}$ Chair and Department of General, Vascular and Oncologic Surgery, $2^{\text {nd }}$ Faculty of Medicine, Warsaw Medical University, Poland \\ ${ }^{2}$ Chair and Department of Obstetrics, Gynecology and Oncology, $2^{\text {nd }}$ Faculty of Medicine, Warsaw Medical University, Poland
}

\begin{abstract}
Background: Unlike other solid tumors (i.e. pancreas, gallbladder, stomach), an ovarian cancer is responsive to a systemic treatment with platinum derivates in $80 \%$ of patients. This apparent chemosensitivity justifies a broader surgical approach. A cytoreductive, "tumor-debulking" surgery is defined as an attempt to remove in a maximum degree all visible and detectable lesions. Despite treatment, the advancement of the disease very often leads to complications defined as "surgical" and life-threatening.

Objectives: The aim was to evaluate the efficacy and safety of palliative surgery in advanced ovarian cancer implicating acute surgical diseases of the abdominal cavity.

Material and methods: Between years 2005 and 2014 were operated 118 patients with an advanced ovarian cancer (FIGO III-IV) implicating acute and directly life-threatening diseases of the abdominal cavity, involving 132 surgical operations. The causes of these operations were: obstruction of the gastrointestinal tract -91 patients; perforation of the gastrointestinal tract - 15; gastrointestinal bleeding - 9; intussusceptions -3 .

Results: Retrospective data for the 118 patients were analyzed. Safety and the perioperative mortality rate were assessed. Serious postoperative complications were recorded in 31 patients (anastomotic stoma -9 ; bleeding requiring repeated surgery -3 ; recurring gastrointestinal obstruction -16 ; liver failure after partial hepatic resection -3 ). Systemic complications in the form of respiratory failure and cardiovascular disorders requiring cardiological treatment -21 . All patients required clinical nutrition, both parenteral and enteral. Deaths recorded -3.39 patients were rehospitalized within 30 days of surgery. 7 deaths were recorded in this group.

Conclusions: Combining lifesaving surgery with cytoreduction allows further adjuvant treatment. Early rehospitalization occurring within less than 30 days is linked to increased mortality.
\end{abstract}

Key words: ovarian cancer, multivisceral resections

Ginekologia Polska 2016; 87, 10: 685-689

\section{INTRODUCTION}

Knowledge about epithelial ovarian cancer (EOC) continuously widened in the last few decades. New theories and discoveries concerning its true origin and particular histological features cast light on EOC's real nature and behavior. The studies aimed to define optimal therapeutic procedures.

While many issues remain unresolved, like an optimal time to perform a primary cytoreductive surgery, the advan- tages resulting from administered drugs, the development of the disease's early detection or even prevention schemes, it is the value of an optimal tumor resection defined by the intensity of the minimal residual disease's relapse that enjoys a well-established position in numerous prospective and retrospective studies. We may read in these studies, that a radical resection of a tumor has a decisive prognostic significance, even in the case of unfavorable factors, such as peritoneal metastasis. While many works exist on the subject of second- 
ary surgical cytoreduction with subsequent chemotherapy, there is a lack of works about cytoreductive surgery in patients undergoing a third or fourth operation complicated by an acute disease of the abdominal cavity. There is only one analysis regarding 15 patients qualified for a quaternary cytoreductive surgery (Memorial Sloan-Kettering Cancer Center (Shih et al., 2010).) The study analyzed the results of surgical treatment and attempted to define the patients for whom such surgery is indicated. In 1983, Berek et al. introduced for the first time the term "secondary cytoreduction". According to most physicians, this is a surgical procedure performed after the completion of basic therapy and intended to reduce the mass of the tumor. This type of surgery aims to improve the patient's quality of life as a result of an elimination of symptoms due to the presence of the tumor. These are non-healing procedures and original studies concerning patients displaying a sub-optimal response to treatment showed only marginal advantages, with a median survival of 9 months [6, 22]. Thus, no proof exists at present that secondary surgery constitutes a real advantage for this population of patients. Ovarian carcinoma increasingly becomes a long-term disease and the issues concerning its treatment at a severely advanced stage involve complications in need of acute care surgery of the abdominal cavity. The usefulness of such procedure was called into question in the case of a selected group of patients, when it appeared that after the elimination of the cause of a gastrointestinal obstruction and the removal of a large and poorly vascularized tumorous mass, the patients began once again to respond well to the adjuvant treatment. A surgical justification for the elimination of the cause, like an obstruction, but also for the removal of the tumor, is a reinforcement of the immune system due to the reduction of the tumor's mass.

\section{MATERIAL AND METHODS}

118 patients operated between 2005 and 2014 because of an ovarian carcinoma implicating an acute abdominal cavity disease. Demographic data are presented in Table 1.

\section{RESULTS}

Retrospective data for 118 patients were analyzed. Safety and mortality during the perioperative period were assessed. Severe surgical postoperative complications were observed in 31 patients [anastomosis - 9; bleeding requiring new surgery - 3; recurrence of gastrointestinal obstruction - 16; pancreatitis after partial resection - 3 (Tab. 2)].

Systemic complications like respiratory failure and cardiovascular disorders requiring cardiologic treatment - 21 (Tab. 3). All patients required parenteral, as well as enteral nutrition. Were recorded 3 deaths.

39 patients were rehospitalized within 30 days of surgery. In this group were recorded 7 deaths.

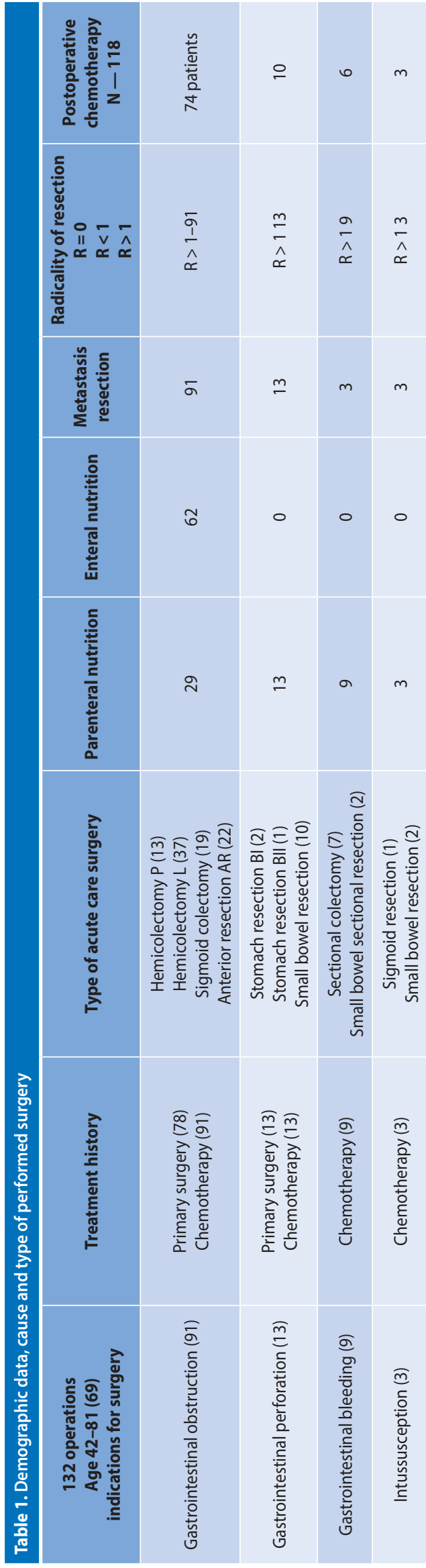


Table 2. Postoperative complications

\begin{tabular}{|l|c|}
\hline Duration of operation (min) & 90-125 (112) \\
\hline Perioperative blood unit transfusion & 36 \\
\hline Transfused blood unit & 9 \\
\hline Anastomotic stoma & 3 \\
\hline Post-operative bleeding & 39 \\
\hline Gastrointestinal obstruction recurrence or occurrence of obstruction as primary symptom of pancreatitis & 3 \\
\hline Pancreatitis & $10-34$ (13) \\
\hline Period of hospitalization (days) & 2 \\
\hline Patients requiring intensive postoperative care (days)
\end{tabular}

Table 3. General medical complications

\begin{tabular}{|c|c|c|c|}
\hline & Perioperative complications & Early postoperative complications & Late postoperative complications \\
\hline \multirow[b]{2}{*}{ Respiratory } & & Exudates (13) & Pneumonia \\
\hline & & Pneumonia (8) & 6 \\
\hline \multirow[b]{2}{*}{ Cardiovascular } & & Myocardial infraction (2) & Circulatory collapse (7) \\
\hline & & & \\
\hline Hematological & Anemia (11) & Anemia (17) & Deep venous thrombosis (2) \\
\hline \multirow[b]{2}{*}{ Intestinal } & & Pancreatitis (2) & lleus (16) \\
\hline & & Anastomotic dehiscence (5) & \\
\hline \multirow{3}{*}{ Infections } & & Abdominal abscess (3) & Abdominal abscess (2) \\
\hline & & Eventration (5) & \\
\hline & & Suppurative lesions (12) & \\
\hline
\end{tabular}

With postoperative surgeon's control 89 patients were embraced (72.9\%). Within 30 days after the surgery 39 patients were rehospitalized due to surgical complications (33\%). Despite intensive treatment, surgical and nutritional 7 patients (aged between 60 and 79) died. For the adjuvant treatment 71 patients from the group under control were classified. They were divided into two parts depending on age. The first group consisted of patients up to 50 years of age and the second one above 50 years of age. To the first group 18 patients were qualified. After a year observation there was no mortality in this group. In the second group there were 53 patients. In this group we observed the annual survival in 31 patients. Annual mortality in the group receiving the adjuvant therapy was $31 \%$.

\section{DISCUSSION}

An analysis of data from 53 studies involving 6885 patients with a $n$ epithelial ovarian carcinoma who were operated to obtain the greatest degree of cytoreduction of lesions and subsequently treated systematically shows, that cytoreduction is an independent prognostic factor related to the rate of survival [1-3]. Each $10 \%$ increase in tumor mass reduction meant a $5.5 \%$ increase of the survival median. Current studies confirm, that an optimal surgical cytoreduction is the most important prognostic factor in case of advanced ovarian carcinoma $[2-5,7,10,17]$. A complete cytoreduction shoud be the aim of every operation, and if this is not possible, the aim should be a minimal residual disease [10-16]. Owing to modern surgical techniques, it is possible to perform a multivisceral resection in patients with a large volume tumor and stage IV disease [5, 17]. Data provided in literature suggest, that women operated by gynecologic oncologists and surgical oncologists have a much greater survival rate compared to the one of patients operated by general gynecologists and surgeons without oncologic instruction [26]. Our material comprises operations performed by a team of oncologic and gynecologic surgeons. This has increased the opportunities to use surgical techniques 
that not only eliminate the cause of a $\mathrm{n}$ acute abdominal disease, but also allow to perform cytoreductive surgery. Also, owing to this, the number of strictly palliative and comfort-reducing operations has been greatly reduced, for example such which are due to stomas. The most frequent complication occurring in a advanced ovarian carcinoma is a gastrointestinal obstruction. It requires emergency surgery - an additional negative postoperative prognostic factor. The rectosigmoid, because of its continuity, is compressed most often and infiltrated by the tumor [18-23]. If this is the unique obstruction-causing section of the gastrointestinal tract, we assume a two-stage procedure. During the first stage, we implant a self-expanding stent to debulk the obstruction and to restore anatomic conditions; then, after 5 to 7 days, we perform a resection. Gastrointestinal debulking by implanting the stent also presents another advantage: the number of debulking stomas has been practically reduced to the situations, where a stent implant is not feasible technically. No large studies exist on the subject of the presented procedural model regarding a gastrointestinal obstruction due to a gynecologic cancer. For example, in a situation where such a complication is lacking, Morton et al. [18] have retrospectively assessed 58 patients who underwent an myometrial resection in conjunction with the rectosygmoid. They restored gastrointestinal continuity in all patients. One patient underwent a colostomy due to an anastomotic leak, and 3 patients underwent pelvic abscess drainage. Similar results were obtained by Peiretti et al. [24], where after a surgical resection due to an ovarian cancer with rectosigmoid infiltration and a restoration of the gastrointestinal continuity in 238 patients, anastomotic leaks occurred in 7 patients, and a pelvic abscesses in 9 . In our material, anastomotic stomas occurred in 9 patients, while the most frequent early complication was a recurrence of intestinal obstruction. It occurred in 39 patients and caused rehospitalization. In this group of patients, in most cases a palliative treatment alleviated obstruction symptoms, however a general emaciation due to malnutrition and and neoplastic cachexia resulted in 7 deaths. In this group of patients, obstruction symptoms were due to a multilevel infiltration of the small intestine and colon. According to Jaeger et al. [28], in case of an advanced carcinoma comprising the intestines, a multilevel resection will not significantly improve the results, despite residual disease resection. However, most authors $[14,20,23,26]$ affirm in their works, that these multivisceral resections indeed improve treatment results in patients. A very difficult group of patients as far as the extent of surgery is concerned, are patients with gastrointestinal perforation and gastrointestinal bleeding. In these groups of patients, surgery - often very extensive and not due to the carcinoma - is extremely taxing and hazardous, but indispensable to save their lives. An optimal cytoreduction in these patients is reserved for a narrow group where the assessment of the operative risk does not exceed ASA II. In our material, 9 patients were operated due to gastrointestinal bleeding. Out of them, 6 qualified for adjuvant therapy. Cytoreduction was performed in 3 patients only. The reason was a preoperative hemorrhagic shock and its possible deepening during cytoreductive surgery.

In our study involving 118 patients, 25 of them (21.2\%) had not qualified for systemic treatment. The most frequent causes were: intestinal obstruction (16 patients); cardiovascular failure (7patients) and massive thrombosis ( 2 patients). 93 patients $(78.8 \%)$ qualified for further treatment. Deaths recorded were 3 (2.5\%). 39 patients were rehospitalized within 30 days. The most frequent cause was neoplastic cachexia, impossibility of oral nutrition (without surgical cause) and symptoms of a cancer-induced sub-obstruction defined as "peritonitis carcinomatosa". Deaths recorded in this group were 7 , that is $18 \%$ of patients.

Summing up, it must be said that an aggressive surgical treatment in respect to patients with an advanced ovarian cancer implicating an acute abdominal disease is a controversial procedure. Controversies center around the lack of a uniform procedural scheme in respect to this group of patients. The traditional surgical procedure consisting only of a local treatment of acute abdominal disease is not an appropriate one in respect to the entire group of patients and it should be reserved for two oncologically opposite groups of patients. The first group comprises patients without residual disease in the abdominal cavity, while the second those patients who display a technical possibility to perform a cytoreduction during emergency surgery. It seems that the extent of surgery in this group of patients is not determined by the advancement of the carcinoma, but by the general condition of the patient, assessed on the basis of the advancement of accompanying internal diseases. This is confirmed by the results of our work, where out of 118 patients, 79 (66.7\%) underwent adjuvant therapy without surgical complications and rehospitalization.

\section{CONCLUSIONS}

Combining lifesaving surgery with cytoreduction provides an opportunity for further adjuvant treatment. Early rehospitalization within 30 days is related to an increased mortality rate.

\section{REFERENCES}

1. Jemal A, Siegel R, Xu J, Ward E. Cancer statistics, 2010. Ca Cancer J Clin. 2010, 60, 277-300.

2. Bristow RE, Tomacruz RS, Armstrong DK, Trimble EL, Montz FJ. Survival effect of maximal cytoreductive surgery for advanced ovarian carcinoma during the platinum era: a meta-analysis. J Clin Oncol. 2002, 20, 1248-1259.

3. Gadducci A, Sartori E, Landoni F, [et al.]. Relationship between time interval from primary surgery to the start of taxane- plus platinum-based chemotherapy and clinical outcome of patie nts with advanced epithelial 
ovarian cancer: results of a multicenter retrospective Italian study. J Clin Oncol. 2005, 23, 751-758.

4. Eisenkop SM, Spirtos NM, Lin WC. "Optimal" cytoreduction for advanced epithelial ovarian cancer: a commentary. Gynecol Oncol. 2006, 103, 329-335.

5. Aletti GD, Dowdy SC, Gostout BS, [et al.]. Aggressive surgical effort and improved survival in advanced-stage ovarian cancer. Obstet Gynecol. $2006,107,77-85$.

6. Hennessy BT, Coleman RL, Markman M. Ovarian cancer. Lancet. 2009, 374, 1371-1382.

7. Elattar A, Bryant A, Winter-Roach B, Hatem M, Naik R. Optimal primary surgical treatment for advanced epithelial ovarian cancer. Cochrane Database Syst Rev. 2011. p. Cd007565.

8. Burger RA, Brady MF, Bookman MA, [et al.]. Incorporation of bevacizumab in the primary treatment of ovarian cancer. N Engl J Med. 2011, 365, 2473-2483.

9. Perren TJ, Swart AM, Pfisterer J, [et al]. A phase 3 trial of bevacizumab in ovarian cancer. N Engl J Med. 2011, 365, 2484-2496.

10. Chang SJ, Bristow RE. Evolution of surgical treatment paradigms for advanced-stage ovarian cancer: redefining 'optimal' residual disease. Gynecol Oncol. 2012, 125, 483-492.

11. Bookman MA. First-line chemotherapy in epithelial ovarian cancer. Clin Obstet Gynecol. 2012, 55, 96-113.

12. Soper JT, Couchman G, Berchuck A, Clarke-Pearson D. The role of partial sigmoid colectomy for debulking epithelial ovarian carcinoma. Gynecol Oncol. 1991, 41, 239-244.

13. Weber AM, Kennedy AW. The role of bowel resection in the primary surgical debulking of carcinoma of the ovary. J Am Coll Surg. 1994, 179, 465-470.

14. Benedetti-Panici P, Maneschi F, Scambia G, Cutillo G, Greggi S, Mancuso $\mathrm{S}$. The pelvic retroperitoneal approach in the treatment of advanced ovarian carcinoma. Obstet Gynecol. 1996, 87, 532-538.

15. Scarabelli C, Gallo A, Franceschi S, [et al.]. Primary cytoreductive surgery with rectosigmoid colon resection for patients with advanced epithelial ovarian carcinoma. Cancer. 2000, 88, 389-397.

16. Hoffman MS, Griffin D, Tebes S, [et al.]. Sites of bowel resected to achieve optimal ovarian cancer cytoreduction: implications regarding surgical management. Am J Obstet Gynecol. 2005, 193, 582-586.

17. Eisenhauer E, Abu-Rustum N, Sonoda Y, [et al.]. The addition of extensive upper abdominal surgery to achieve optimal cytoreduction improves survival in patients with stages IIIC-IV epithelial ovarian cancer. Gynecol Oncol. 2006, 103, 1083-1090.
18. Mourton SM, Temple LK, Abu-Rustum NR, [et al.]. Morbidity of rectosigmoid resection and primary anastomosis in patients undergoing primary cytoreductive surgery for advanced epithelial ovarian cancer. Gynecol Oncol. 2005, 99, 608-614.

19. Estes J, Leath C III, Straughn JM Jr, [et al.]. Bowel resection at the time of primary debulking for epithelial ovarian carcinoma: outcomes in patients treated with platinum and taxane-based chemotherapy. J Am Coll Surg. 2006, 203 (4), 527-532.

20. Park JY, Seo SS, Kang S, [et al.]. The benefits of low anterior en bloc resection as part of cytoreductive surgery for advanced primary and recurrent epithelial ovarian cancer patients outweigh morbidity concerns. Gynecol Oncol. 2006, 103, 977-984.

21. Aletti GD, Podratz KC, Jones MB, Cliby WA. Role of rectosigmoidectomy and stripping of pelvic peritoneum in outcomes of patients with advanced ovarian cancer. J Am Coll Surg. 2006, 203, 521-526.

22. Zivanovic $\mathrm{O}$, Eisenhauer $\mathrm{E}$, Zhou Q, [et al.].The impact of bulky upper abdominal disease cephalad to the greater omentum on surgical outcome for stage IIIC epithelial ovarian, fallopian tube, and primary peritoneal cancer. Gynecol Oncol. 2008, 108, 287-292.

23. Gallotta V, Fanfani F, Vizzielli G, [et al.]. Douglas peritonectomy compared to recto-sigmoid resection in optimally cytoreduced advanced ovarian cancer patients: analysis of morbidity and oncological outcome. Eur J Surg Oncol. 2011, 37, 1085-1092.

24. Peiretti M, Bristow R, Zapardiel I, [et al.]. Rectosigmoid resection at the time of primary cytoreduction for advanced ovarian cancer. A multi-center analysis of surgical and oncological outcomes. Gynecol Oncol. 2012, 126, 220-223.

25. Angioli R, Plotti F, Aloisi A, [et al.]. Does extensive upper abdomen surgery during primary cytoreduction impact on long-term quality of life? Int J Gynecol Cancer. 2013, 23, 442-447.

26. Paulsen T, Kjaerheim K, Kaern J, Tretli S, Trope C. Improved short-term survival for advanced ovarian, tubal, and peritoneal cancer patients operated at teaching hospitals. Int J Gynecol Cancer. 2006, 16 (Suppl 1), 11-17.

27. Gillette-Cloven N, Burger R, Monk B, [et al.]. Bowel resection at the time of primary cytoreduction for epithelial ovarian cancer. J Am Coll Surg. 2001, 193, 626-632. [

28. JaegerW, Ackermann S, Kessler H, Katalinic A, Lang N. The effect of bowe resection on survival in advanced epithelial ovarian cancer. Gynecol Oncol. 2001, 83, 286-291. 